Spatially translatable optical fiber-coupled heterodyne interferometer

Byonghoon Seo, and Paul M. Bellan

Citation: Review of Scientific Instruments 88, 123504 (2017);

View online: https://doi.org/10.1063/1.5007070

View Table of Contents: http://aip.scitation.org/toc/rsi/88/12

Published by the American Institute of Physics

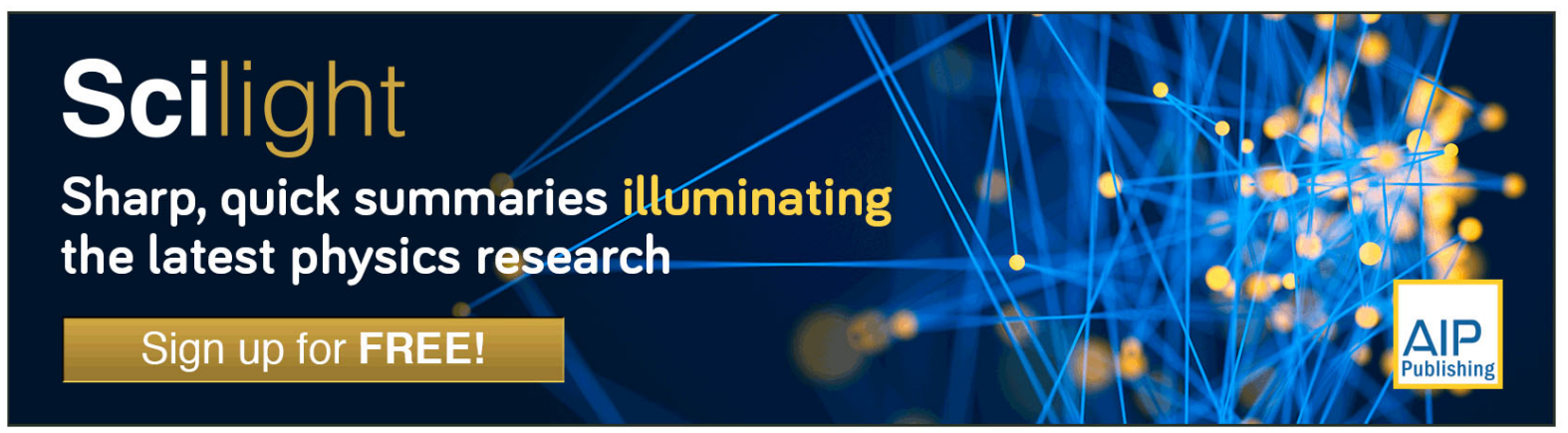




\title{
Spatially translatable optical fiber-coupled heterodyne interferometer
}

\author{
Byonghoon Seo and Paul M. Bellan \\ Applied Physics and Materials Science, Caltech, Pasadena, California 91125, USA
}

(Received 29 September 2017; accepted 20 November 2017; published online 13 December 2017)

\begin{abstract}
An interferometer is a useful diagnostic tool for measuring line-averaged electron density but is limited in its use because it generally measures at a fixed location. We report here a spatially translatable fiber-coupled interferometer that measures the density of a high-speed MHD-driven plasma jet colliding with a target cloud. The interferometer uses a He-Ne laser coupled to a polarization-maintaining single mode optical fiber having a vacuum feedthrough. The interferometer provides a measure of the spatial-temporal profile of the line-averaged electron density from which the change in jet velocity as a result of its collision with the target cloud can be deduced. Published by AIP Publishing. https://doi.org/10.1063/1.5007070
\end{abstract}

\section{INTRODUCTION}

Laser interferometers are commonly used to measure the line-averaged electron density ${ }^{1-5}$ of laboratory plasmas. The interferometer measures a plasma-induced phase shift from which the line-averaged electron density is deduced using a presumed laser beam path length through the plasma. Because the interferometer requires only a low-level signal, a continuous laser can be used so the density time-dependence is measured. An example of such an interferometer is given in Refs. 5 and 6 where the density of a $50 \mathrm{~km} / \mathrm{s}$ MHD driven jet was measured over tens of microseconds with sub-microsecond time resolution. In contrast, Thomson scattering requires a pulsed laser and so measures density at a single time only; however, this is a point measurement rather than a line-averaged measurement. Thomson scattering apparatus is much more complicated and expensive than a laser interferometer.

Besides being limited to a line-averaged measurement, another drawback of standard laser interferometer systems is that the laser beam location is fixed ${ }^{1,3,4}$ so that measurement of the spatial dependence of the density is not possible unless multiple laser beams are used as, for example, in the multichord fiber-coupled system described in Ref. 3. While a multichord system provides spatial resolution, it increases the cost and complexity of the optical system and the spatial resolution is limited by the number of chords used.

We describe here a single-chord interferometer that provides arbitrary spatial resolution. This interferometer was developed to measure the increase in electron density that occurs when an MHD-driven magnetized plasma jet collides with a target gas cloud. The purpose of this measurement is to investigate the scaling of the adiabatic compression proposed for magnetized target fusion. ${ }^{7-9}$ The collision in the experiment simulates the compression of magnetized plasma resulting from an imploding liner. The simulation involves a change of frames where the stationary target cloud represents the imploding liner and the MHD-driven plasma jet represents the plasma being compressed by the liner. Because the location where the collision occurs varies depending on initial conditions, it is desirable to have an interferometer that can measure the time-dependent density at an arbitrary location.
The spatially translatable heterodyne interferometer described here uses a polarization-maintaining, single-mode optical fiber mounted on a vacuum feedthrough. By adjusting the location of the interferometer beam in the vacuum chamber, the spatial profile of the line-averaged electron density is measured. The multi-point spatial-temporal measurement reveals not only a density increase when the jet impacts the target cloud but also a quantitative measurement of the substantial reduction in jet velocity when this impact occurs.

\section{EXPERIMENTAL SETUP}

The experiment is performed in the $1.4 \mathrm{~m}$ diameter, $1.6 \mathrm{~m}$ long cylindrical vacuum chamber shown in Fig. 1.,10 The chamber is much larger than the jet or the cloud so there are negligible plasma interactions with the chamber wall. A Cartesian coordinate system $\{x, y, z\}$ is used with the $z$ axis along the direction of the symmetry axis of the cylindrical vacuum chamber; this is also the direction of the jet flow. The $z$ direction will also be referred to as the axial direction. The term "poloidal" will be used to refer to the $r$ and $z$ directions of a magnetic field as conventionally used in cylindrical coordinates.

The jet ${ }^{6,10,11}$ originates at concentric coplanar disk and annulus electrodes; the electrode axial position defines $z=0$. The disk electrode is $20 \mathrm{~cm}$ diameter, and the annulus electrode inner diameter is slightly larger than $20 \mathrm{~cm}$ so there is a gap between the electrodes; the annulus outer diameter is $51 \mathrm{~cm}$. A bias coil behind the disk electrode creates an initial dipolelike poloidal magnetic field that links the disk and annulus electrodes. Neutral hydrogen is puffed into the vacuum chamber from 8 gas nozzles on the disk electrode and from 8 nozzles on the annulus electrode. A capacitor bank charged to a nominal $4.5 \mathrm{kV}$ breaks down the hydrogen gas to form plasma. Initially, 8 plasma loops are formed each of which spans from a nozzle in the disk to a nozzle in the annulus. These loops form along the initial poloidal magnetic field and reveal the dipole shape of the initial field. The collection of 8 loops has a shape similar to the 8 legs of a spider. ${ }^{11}$ The radially inner parts of the 8 spider legs merge to form the MHD jet. This jet propagates in the $z$ direction away from the electrodes and the jet 


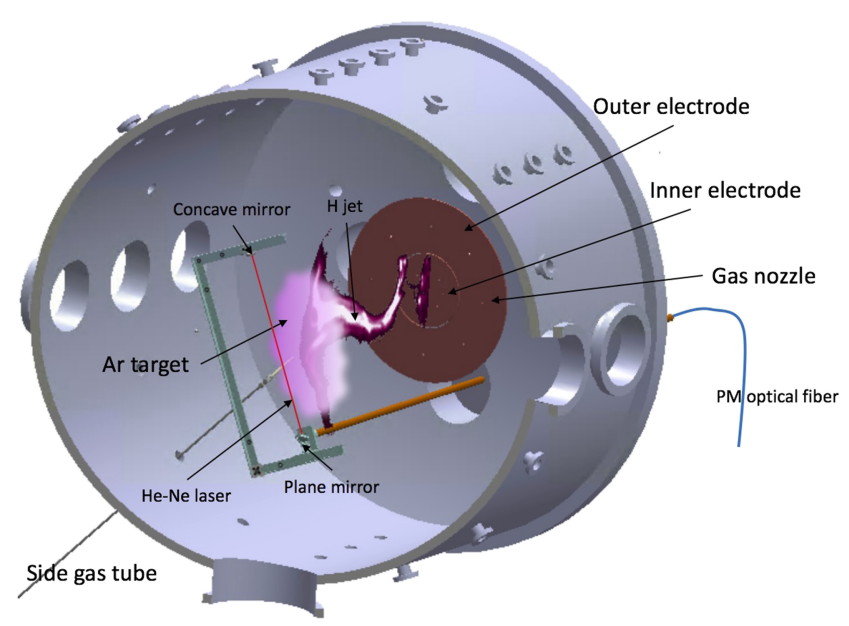

FIG. 1. Schematic of the vacuum chamber, PM: polarization-maintaining single-mode optical fiber.

self-collimates via MHD forces. ${ }^{12}$ The target cloud consists of argon gas puffed from a side tube ${ }^{13}$ located at $z=370 \mathrm{~mm}$. The jet collides with and ionizes the argon cloud. There is a large difference between the jet velocity and that of the argon cloud. The jet is accelerated to $\sim 50 \mathrm{~km} / \mathrm{s}$ by MHD forces, whereas the argon cloud expands at the $0.25 \mathrm{~km} / \mathrm{s}$ thermal velocity of room temperature argon. Thus, the argon cloud can be considered stationary relative to the jet, and the $z$ location of the jet-cloud collision can be controlled via the timing of the argon gas cloud puff valve.

The hardware providing the axially translatable interferometer beam is sketched in Figs. 1 and 2. This hardware consists of an optical fiber located inside a $19 \mathrm{~mm}$ stainlesssteel tube (shown as orange in Figs. 1 and 2), a U-shape supporting structure (shown as green in Fig. 1), a plane mirror, and a concave mirror. The fiber ${ }^{14}$ is single-mode and polarization-maintaining with a $4 / 125 \mu \mathrm{m}$ core/cladding diameter. A multi-mode fiber was used in an initial attempt to construct the interferometer, but this did not work because the multi-mode configuration greatly limited the extent to which the beam could be collimated. This insufficient collimation resulted in substantial beam divergence so the signal became

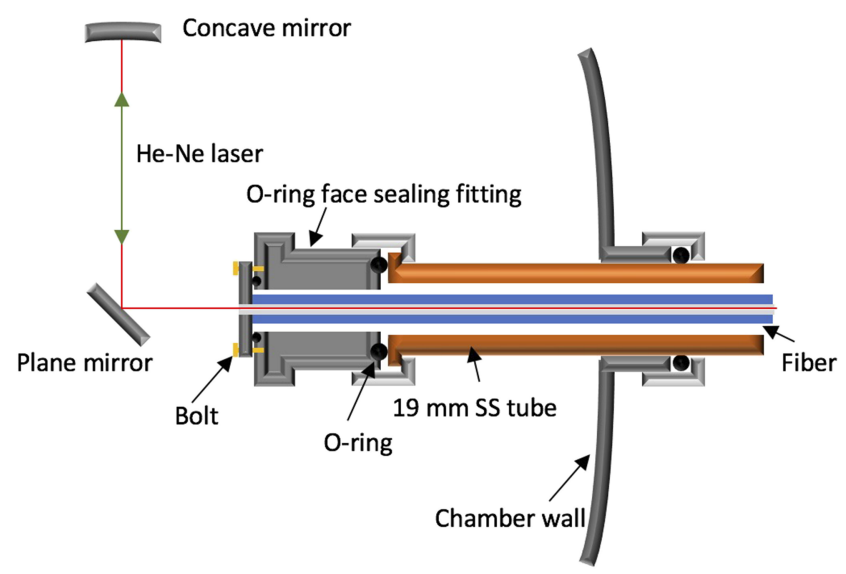

FIG. 2. Schematic of the vacuum compatible optical fiber and the vacuum feedthrough (not scaled). too weak. Polarization maintenance turned out to be critical because the signal intensity depends on the polarization and jumps in polarization of a non-polarization maintaining fiber adversely affected the signal. The optical fiber couples to vacuum at the end of the $19 \mathrm{~mm}$ stainless steel tube via an O-ring sealed vacuum feedthrough located at the left end of the orange tube sketched in Fig. 2. The $25 \mathrm{~mm}$ plane mirror is installed at the bottom of the $\mathrm{U}$-shape structure using vacuum-compatible mirror mounts. ${ }^{15}$ This mirror is at $45^{\circ}$ so as to deflect the beam by $90^{\circ}$ so that the deflected beam propagates perpendicular to the $z$ axis.

The beam propagates through the plasma and then reflects from the concave mirror mounted at the top of the U-shaped structure. The reflected beam bounces off the $45^{\circ}$ mirror and reenters the optical fiber. Thus, the interferometer beam makes a double-pass through the plasma, first going up and then going down. Initial attempts to construct the interferometer used a retroreflector instead of the concave mirror, but it was found that in this case the signal was weak because the divergence of the reflected light greatly reduced the amplitude of the reflected beam. This problem could not be alleviated by using a collimator. The concave mirror allows the beam to be slightly divergent on exiting the fiber because the mirror focuses the beam back onto the face of the fiber, so the divergence is effectively removed. The focal length of the concave mirror is $300 \mathrm{~mm}$, and the distance from the optical fiber to the concave mirror is $600 \mathrm{~mm}$ so the concave mirror produces a one-toone image of the fiber face onto itself. Fine-centering of the reflected beam onto the fiber is accomplished by adjusting the tilt angle of the concave mirror.

The laser beam was axially translated by axial motion of the $19 \mathrm{~mm}$ stainless tube so that measurements could be made at different values of $z$. The $19 \mathrm{~mm}$ stainless tube entered the vacuum chamber through an O-ring sealed vacuum quick disconnect that enabled axial motion. There was additional mechanical support of the tube on the atmosphere side to ensure straight motion in the $z$ direction. The $19 \mathrm{~mm}$ stainless tube could also be rotated about its translation axis so the laser beam could traverse different cross sections of the plasma.

An initial design used a U-shaped structure constructed of steel tubes. However, it was found that when the $19 \mathrm{~mm}$ stainless tube was rotated about its axis, the U-shaped structure would deform enough to cause a substantial change in the interferometer phase and amplitude. The change in amplitude was presumed to result from a misalignment of the reflected laser beam as consequence of a gravity-caused shift in the location of the concave mirror relative to the rest of the optics. To eliminate this gravitational problem, the U-shaped structure was reconstructed from T-shaped aluminum channel and stiffened with diagonal plates.

The remainder of the interferometer consists of a $\mathrm{He}-\mathrm{Ne}$ laser, an optical isolator, an acoustic optic modulator, beam splitters, mirrors, and a detector installed on an optical table as described in Ref. 5. The opposite end of the $10 \mathrm{~m}$ long fiber was brought to this table and was mounted on an optical fiber launcher ${ }^{16}$ located on the optical table as shown in Fig. 3. The laser beam splits into two paths: one is the scene beam (beam going into the fiber and then coming back after double 


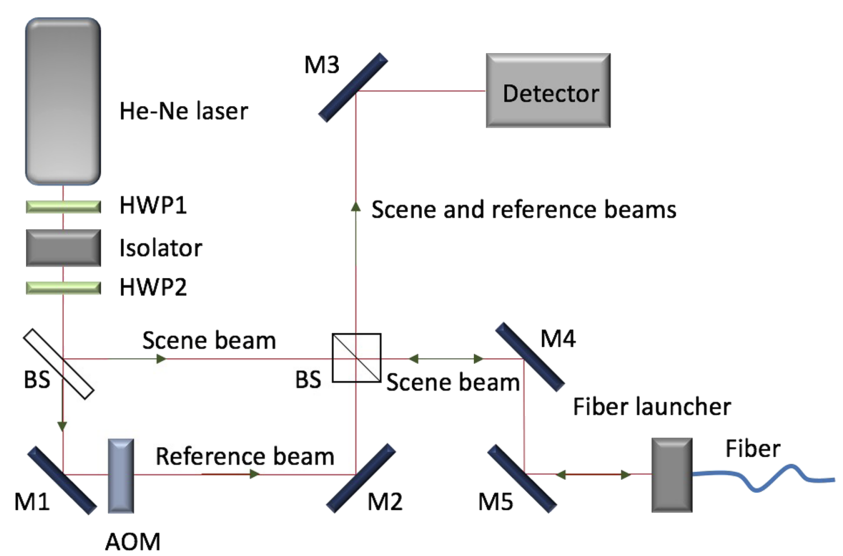

FIG. 3. Schematic of optics on the optical table for the interferometer, M1 M5: silver mirrors, BS: beam splitter, HWP: half wave plate, AOM: acoustic optic modulator with $80 \mathrm{MHz}$.

passing through the plasma), and the other is the reference beam. As discussed in Ref. 5, the reference beam length is short, about $45 \mathrm{~cm}$. The fiber launcher and mirrors M4 and M5 were adjusted to guide the light from the laser into the fiber with $\sim 40 \%$ transmittance efficiency.

The scene beam exiting the fiber reflected from the beam splitter (BS) toward the detector. Mirror M2 and BS were adjusted to superimpose the scene and reference beams. Mirror M3 was adjusted to center the superimposed beams on the detector. The reference beam is modulated by an $80 \mathrm{MHz}$ RF-driven acousto-optic modulator.

To summarize, the scene beam coming from the laser enters the fiber at the fiber end on the optical table and exits the other end of the fiber at the vacuum feedthrough at the end of the $19 \mathrm{~mm}$ stainless tube. The scene beam then reflects from the $45^{\circ}$ plane mirror, traverses the plasma, reflects from the concave mirror, traverses the plasma again, reflects again from the $45^{\circ}$ plane mirror, re-enters the fiber, and then exits the fiber at the optical table. The double pass of the scene beam makes the mechanical setup sufficiently simple and robust to enable axial translation and rotation of the scene beam while maintaining optical alignment. The $80 \mathrm{MHz}$ signal from the mixing of the scene and reference beams on the detector is amplified and split into two by an RF power divider. One of these two signals is mixed with an $80 \mathrm{MHz}$ "cosine" reference signal and the other with an $80 \mathrm{MHz}$ "sine" reference signal to obtain quadrature phasing. The cosine signal is a sample of the original $80 \mathrm{MHz}$ signal used to drive the acousto-optic modulator while the sine signal is obtained by phase-shifting this sample by $90^{\circ} .5$

\section{RESULTS}

Figure 4 shows the interferometer quadrature signals from a typical plasma shot. The time at which the plasma jet intercepts the beam is indicated.

The plasma-induced phase change $\Delta \phi_{p}$ can be deduced from taking the inverse tangent of the ratio of the sine and cosine signals. The electron density is then obtained assuming that the plasma is centered on the $z$ axis and using

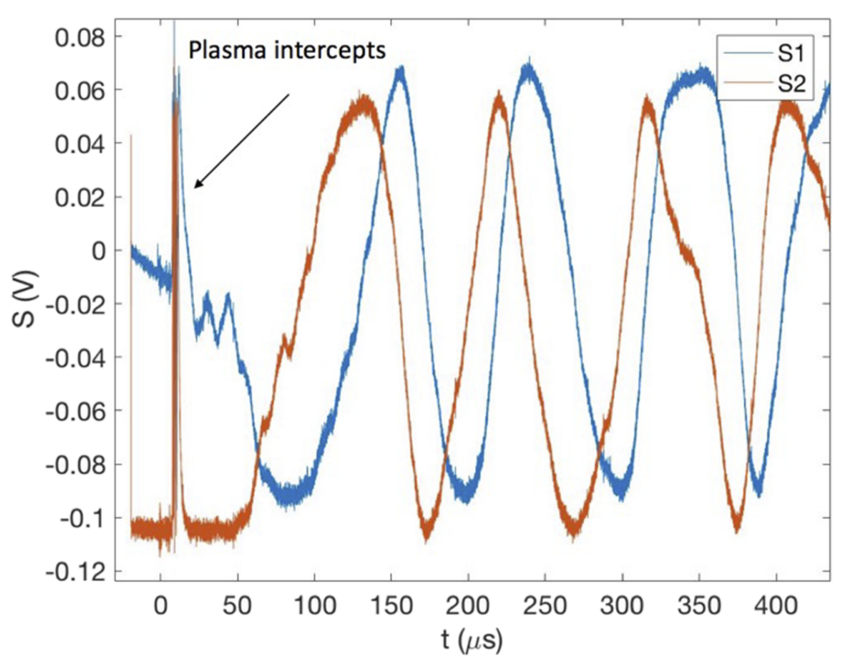

FIG. 4. Quadrature signals.

$$
\Delta \phi_{p}=\frac{e^{2} \lambda_{0}}{4 \pi c^{2} m_{e} \epsilon_{0}} \times 2 \int_{-L / 2}^{L / 2} n(x, y, z) d x,
$$

where $c$ is speed of light, $m_{e}$ is the electron mass, $\epsilon_{0}$ is the permittivity of free space, $e$ is the electron charge, $L=0.6$ $\mathrm{m}$ is the beam traverse of the plasma, and $\lambda_{0}=632.8 \mathrm{~nm}$ is the He-Ne laser wavelength. Figure 5 shows a measured timedependent density trace averaged over 3 shots when the MHDdriven hydrogen plasma jet collides with the argon target cloud at $z=300 \mathrm{~mm}$. A line-averaged electron density $8 \times 10^{21} \mathrm{~m}^{-3}$ is measured. The very small shot-to-shot scatter shows that the shots are sufficiently reproducible for axial scanning of the interferometer position over a sequence of shots to be a feasible method for determining the axial dependence of the line-averaged plasma density.

Figure 6 shows electron densities obtained by translating the interferometer in the $z$ direction for a sequence of identical shots where the hydrogen plasma jet collides with the argon target cloud. The color map indicates electron density (calibration by color bar on the side), and the asterisk indicates the maximum density in the time-dependent density traces.

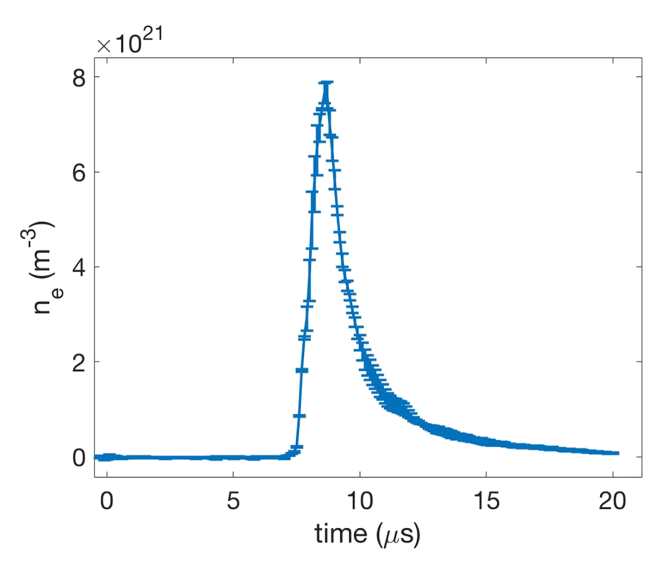

FIG. 5. Electron density trace in time with 3 shots averaged from shot 19528 to 19530 . The errorbar indicates the standard deviation of the data taken over 3 shots. 


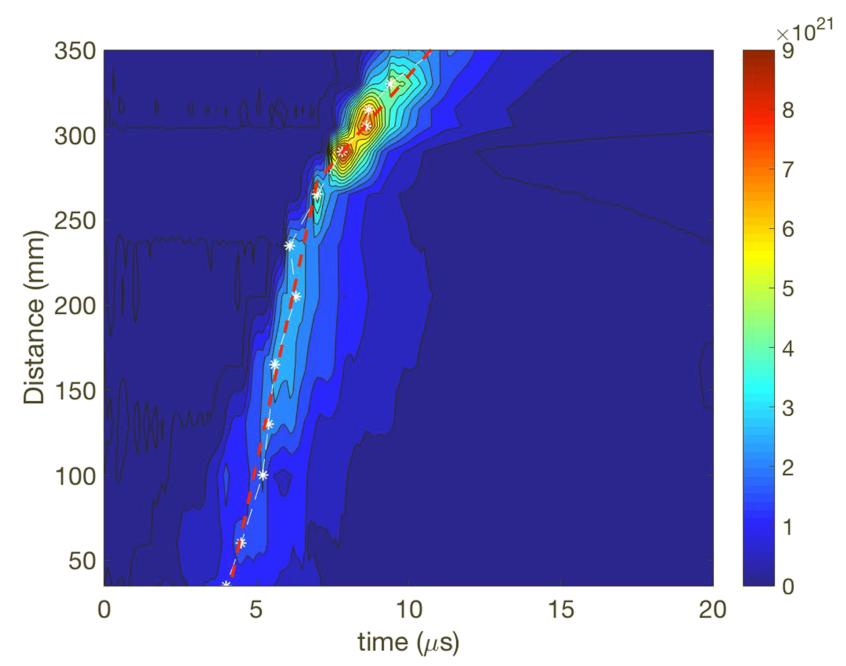

FIG. 6. $n_{e}$ measured by translating the interferometer from shots 19510 to 19611. Asterisks indicate maximum densities at each location. The red dashed line is fitted using a linear function.

The horizontal axis is time, and the vertical axis is the $z$ distance from the electrode. The peak density is $9 \times 10^{21} \mathrm{~m}^{-3}$ at $z \simeq 280 \mathrm{~mm}$ and $t \simeq 7.5 \mu \mathrm{s}$. The experiment was optimized for the jet and gas cloud to collide at $280 \mathrm{~mm}$; this optimization was achieved by varying the timing of the argon gas puff valve so as to control the location of the argon cloud. Since the density rise-time is fast (see Fig. 5) and Fig. 6 plots distance versus time, the slope of the asterisks in Fig. 6 indicates the velocity of the jet front. It is seen that the slope changes at $\sim 280 \mathrm{~mm}$ when the jet collides with the target cloud. Linear fitting to the slope before and after the collision shows that the jet velocity changes from $\sim 70 \mathrm{~km} / \mathrm{s}$ before collision (i.e., at $z$ $<280 \mathrm{~mm}$ ) to $\sim 20 \mathrm{~km} / \mathrm{s}$ after collision (i.e., at $z>280 \mathrm{~mm}$ ). The slopes are indicated by the red dashed lines in Fig. 6. The increase in jet density and the decrease in jet velocity clearly show that the MHD-driven plasma jet is compressed when it impacts the argon cloud.

\section{CONCLUSION}

We have shown here a spatially translatable fiber-coupled interferometer that measures the density of a high-speed MHD-driven plasma jet colliding with a target cloud. The interferometer uses a He-Ne laser coupled to a polarizationmaintaining single mode optical fiber having a vacuum feedthrough. The interferometer provides a measure of the spatial-temporal profile of the line-averaged electron density from which the change in jet velocity as a result of its collision with the target cloud can be deduced.

\section{ACKNOWLEDGMENTS}

Supported by USDOE ARPA-E Grant No. DEAR0000565.

${ }^{1}$ S. Okajima, K. Nakayama, H. Tazawa, K. Kawahata, K. Tanaka, T. Tokuzawa, Y. Ito, and K. Mizuno, Rev. Sci. Instrum. 72, 1094 (2001).

${ }^{2}$ H. Lamela, P. Acedo, and J. Irby, Rev. Sci. Instrum. 72, 96 (2001).

${ }^{3}$ E. C. Merritt, A. G. Lynn, M. A. Gilmore, and S. C. Hsu, Rev. Sci. Instrum. 83, 033506 (2012).

${ }^{4}$ L. M. Smith, D. R. Keefer, and N. W. Wright, Rev. Sci. Instrum. 74, 3324 (2003).

${ }^{5}$ D. Kumar and P. M. Bellan, Rev. Sci. Instrum. 77, 083503 (2006).

${ }^{6}$ D. Kumar and P. M. Bellan, Phys. Rev. Lett. 103, 105003 (2009).

${ }^{7}$ I. R. Lindemuth and R. E. Siemon, AIP Conf. Proc. 1154, 68 (2009).

${ }^{8}$ M. R. Gomez, S. A. Slutz, A. B. Sefkow, D. B. Sinars, K. D. Hahn, S. B. Hansen, E. C. Harding, P. F. Knapp, P. F. Schmit, C. A. Jennings et al., Phys. Rev. Lett. 113, 155003 (2014).

${ }^{9}$ S. A. Slutz, M. C. Herrmann, R. A. Vesey, A. B. Sefkow, D. B. Sinars, D. C. Rovang, K. J. Peterson, and M. E. Cuneo, Phys. Plasmas 17, 056303 (2010).

${ }^{10}$ S. C. Hsu and P. M. Bellan, Phys. Plasmas 12, 032103 (2005).

${ }^{11}$ S. You, G. S. Yun, and P. M. Bellan, Phys. Rev. Lett. 95, 045002 (2005).

${ }^{12}$ P. M. Bellan, Fundamentals of Plasma Physics (Cambridge, 2008).

${ }^{13}$ A. L. Moser and P. M. Bellan, Astrophys. Space Sci. 337, 593 (2012).

${ }^{14}$ See www.OZoptics.com for OZ Optics, Custom-made polarizationmaintaining single mode optical fiber, Part \#: LPSC-03-633-4/125-P3.2GR-11-40-1A-3-10+LPC-03-0.6-3.2GR-40-SP.

${ }^{15}$ See www.thorlabs.com for Thorlabs, Polaris mirror mounts.

${ }^{16}$ See www.newport.com/p/9095 for Newport, Optical fiber launcher, Part \#: 9095. 